\title{
Strain-specific diversity of mucus-binding proteins in the adhesion and aggregation properties of Lactobacillus reuteri
}

Correspondence

Donald MacKenzie donald.mackenzie@bbsrc.ac.uk Nathalie Juge nathalie.juge@bbsrc.ac.uk

Received 6 July 2010

Revised 9 September 2010

Accepted 10 September 2010

\author{
Donald A. MacKenzie, ${ }^{1}$ Faye Jeffers, ${ }^{1}$ Mary L. Parker, ${ }^{1}$ \\ Amandine Vibert-Vallet, ${ }^{1}$ Roy J. Bongaerts, ${ }^{1}$ Stefan Roos, ${ }^{2}$ Jens Walter $^{3}$ \\ and Nathalie Juge ${ }^{1}$ \\ ${ }^{1}$ Institute of Food Research, Norwich Research Park, Colney, Norwich NR4 7UA, UK \\ ${ }^{2}$ Department of Microbiology, Swedish University of Agricultural Sciences, S-750 07 Uppsala, \\ Sweden \\ ${ }^{3}$ Department of Food Science and Technology, University of Nebraska, Lincoln, NE, USA
}

\begin{abstract}
Mucus-binding proteins (MUBs) have been revealed as one of the effector molecules involved in mechanisms of the adherence of lactobacilli to the host; mub, or mub-like, genes are found in all of the six genomes of Lactobacillus reuteri that are available. We recently reported the crystal structure of a Mub repeat from L. reuteri ATCC 53608 (also designated strain 1063), revealing an unexpected recognition of immunoglobulins. In the current study, we explored the diversity of the ATCC 53608 mub gene, and MUB expression levels in a large collection of $L$. reuteri strains isolated from a range of vertebrate hosts. This analysis revealed that the MUB was only detectable on the cell surface of two highly related isolates when using antibodies that were raised against the protein. There was considerable variation in quantitative mucus adhesion in vitro among $L$. reuteri strains, and mucus binding showed excellent correlation with the presence of cell-surface ATCC 53608 MUB. ATCC 53608 MUB presence was further highly associated with the autoaggregation of $L$. reuteri strains in washed cell suspensions, suggesting a novel role of this surface protein in cell aggregation. We also characterized MUB expression in representative L. reuteri strains. This analysis revealed that one derivative of strain 1063 was a spontaneous mutant that expressed a C-terminally truncated version of MUB. This frameshift mutation was caused by the insertion of a duplicated $13 \mathrm{nt}$ sequence at position $4867 \mathrm{nt}$ in the mub gene, producing a truncated MUB also lacking the C-terminal LPxTG region, and thus unable to anchor to the cell wall. This mutant, designated 1063N (mub-4867), displayed low mucusbinding and aggregation capacities, further providing evidence for the contribution of cell-wallanchored MUB to such phenotypes. In conclusion, this study provided novel information on the functional attributes of MUB in L. reuteri, and further demonstrated that MUB and MUB-like proteins, although present in many $L$. reuteri isolates, show a high genetic heterogeneity among strains.
\end{abstract}

\section{INTRODUCTION}

The adult human intestine hosts $10^{13}$ to $10^{14}$ bacteria belonging to at least 500 different species or strains (Dethlefsen et al., 2008). Up to nine different bacterial

Abbreviations: EPS, exopolysaccharide(s); FCM, flow cytometry; FSC, forward scatter; GIT, gastrointestinal tract; LaCOG, Lactobacillalesspecific clusters of orthologous protein coding genes; MCM, mouse colonic mucus; MWCO, molecular weight (mass) cut-off; MUB, mucusbinding protein; PI, propidium iodide; PMT, photomultiplier tube; qRTPCR, quantitative real-time PCR; SSC, side scatter.

Two supplementary figures and two supplementary tables are available with the online version of this paper. phyla are usually found; however, the Firmicutes and Bacteroidetes account for over $90 \%$ of all bacteria in the gut (Ley et al., 2008). Despite the striking conservation at a higher phylogenetic level, the abundance of bacteria at species or strain level varies extensively between nonrelated individuals. Nevertheless, a core gut microbiome that is shared among different individuals ensures conservation of the metabolic functions provided by the microbiota (Turnbaugh et al., 2009). It is assumed that this intricate association required significant coevolution of the host and its microbiota, guided by positive selection for factors that result in a state of both mutual tolerance and benefit. The Gram-positive bacterium Lactobacillus reuteri 
is an excellent model organism to study the evolutionary mechanisms of a vertebrate gut symbiont and to test the hypothesis of coevolution, as this species stably inhabits the gastrointestinal tract (GIT) of mammals as diverse as humans, pigs, mice and rats as well as different species of birds (Oh et al., 2010). L. reuteri is one of the dominant species in the GIT of rodents, pigs and chickens (Leser et al., 2002; Salzman et al., 2002; Brooks et al., 2003; Abbas Hilmi et al., 2007) and is considered autochthonous to the human gut in certain individuals, although it is not detectable in most human subjects (Reuter, 2001; Walter et al., 2001; Heilig et al., 2002; Walter, 2008). L. reuteri is widely documented to benefit humans and animals (Connolly, 2009). The phylogenetic relationships reported by Oh et al. (2010) suggest that the beneficial attributes of $L$. reuteri strains could be the outcome of a long-term evolutionary process that resulted in a mutualistic relationship between microbe and host.

A possible mechanism for bacterial adherence and colonization of the host involves the binding of microbial cell-surface molecules to the protective mucus layer covering the epithelial cells of the GIT. An example is provided by the distribution of mucus-binding proteins (MUB) encoded by Lactobacillales-specific clusters of orthologous protein coding genes (LaCOG) in Lactobacillus genomes (Kleerebezem et al., 2010). In total, the 47 proteins with one or more Mub repeat found in the exoproteomes of six Lactobacillus genomes are distributed over six separate LaCOG. The largest cluster, LaCOG 01470, encodes 14 proteins that possess either the MucBP (Pfam PF06458) domain, the recently defined extended Mub repeat (Boekhorst et al., 2006; Mackenzie et al., 2009), or combinations of both domains. These Mub-repeat-containing proteins are most abundant in lactobacilli of the GIT, strongly suggesting that the Mub repeat is a functional unit that could fulfil an important function in host-microbe interactions. Most of the proteins with multiple Mub repeats contain, in addition to a signal peptide, a C-terminal anchoring motif called LPxTG, which is recognized by a family of enzymes called sortases for covalent attachment to the peptidoglycan of the bacterial cell wall (Navarre \& Schneewind, 1999).

The extracellular $353 \mathrm{kDa}$ MUB from L. reuteri 1063 (ATCC 53608) contains two types of related amino acid repeats (Mub1 and Mub2); six copies (RI-RVI) of the type 1 repeat (Mub1) and eight copies (R1-R8) of the type 2 repeat (Mub2) (Roos \& Jonsson, 2002). The six Mub1 repeats are rather diverse (31-87\% aa identity) whereas the Mub2 repeats show very low sequence variation $(83-100 \%$ aa identity). We recently reported the first three-dimensional structure of a type 2 Mub repeat from the L. reuteri 1063 (ATCC 53608) MUB, providing insight into a previously undetected immunoglobulin (Ig)-binding activity for the repeat structural unit of MUBs (MacKenzie et al., 2009).

Interestingly, sequences corresponding to MucBP domains are present in all the currently available genomes of sequenced L. reuteri strains (100-23, DSM 20016 ${ }^{\mathrm{T}}$, MM2-3,
MM4-1, ATCC 55730 and CF48-3A), suggesting an important role in the evolution of this species. However, little is known about the diversity of MUBs in L. reuteri and their contribution to phenotypes. In this study, we investigated the mucus-binding properties of a large collection of $L$. reuteri strains isolated from a range of vertebrate hosts and correlated the degree of adherence of a subset of strains to the presence and expression of MUB to gain an understanding of the evolution of these proteins and their effect on the adhesion properties of this vertebrate symbiont.

\section{METHODS}

Strains, media and growth conditions. The strains of $L$. reuteri used in this study are listed in Table 1. Pure cultures were obtained by streaking out for single colonies on modified MRS (mMRS) agar, as described by $\mathrm{Oh}$ et al. (2010), after anaerobic growth in $5 \%(\mathrm{v} / \mathrm{v})$ $\mathrm{CO}_{2}, 10 \%(\mathrm{v} / \mathrm{v}) \mathrm{H}_{2}$ and $85 \%(\mathrm{v} / \mathrm{v}) \mathrm{N}_{2}$ at $37^{\circ} \mathrm{C}$ for 3-4 days. Liquid cultures were incubated without shaking at $37{ }^{\circ} \mathrm{C}$ in $20 \mathrm{ml}$ standard MRS broth $(2 \%, w / v$, glucose $)$ for up to $24 \mathrm{~h}$ depending on the

Table 1. L. reuteri strains

\begin{tabular}{|ll|}
\hline Strain $^{*}$ & Host \\
\hline ATCC 53608 & Pig \\
DSM 20016 & T \\
MM4-1a & Human \\
MF2-3 & Human \\
CF4-6g & Human \\
FJ1 & Human \\
MF14-C & Human \\
LMS11-3 & Human \\
sr11 & Human \\
1063 N $\dagger$ & Human \\
JW2015 & Pig \\
Lp167-67 & Pig \\
20-2 & Pig \\
3 c6 & Pig \\
CR & Pig \\
One-One & Rat \\
ATCC 55739 & Rat \\
DSM 17509 $(=100-23)$ & Rat \\
N2D & Rat \\
R2LC & Rat \\
ML1 & Rat \\
\#20 & Mouse \\
r13 & Mouse \\
Lr4020 & Mouse \\
LB54 & Mouse \\
\hline
\end{tabular}

${ }^{*}$ All strains were kindly supplied by Jens Walter, University of Nebraska, USA, except for ATCC 53608, which came from the ATCC, and $1063 \mathrm{~N}$, which is a derivative of 1063 (this study). For further information on host and provenance please refer to Oh et al. (2010). $\dagger 1063 \mathrm{~N}$ is a spontaneous mutant containing the $m u b-4867^{\mathrm{i}}$ insertion described in this paper. 
application. Strains were maintained as frozen stocks held at $-80{ }^{\circ} \mathrm{C}$ in MRS broth containing $50 \%(\mathrm{v} / \mathrm{v})$ glycerol.

Production of anti-MUB antibodies. Purified recombinant MubR5 from L. reuteri ATCC 53608 was prepared as described previously (MacKenzie et al., 2009). Polyclonal anti-MUB antibodies were raised in rabbits by BioGenes to a titre of $>1: 200000$. The specificity of the antibody was tested by ELISA against recombinant Mub repeats from L. reuteri ATCC 53608 and BSA as negative control. Microtitre plate wells were coated with target protein $\left(10 \mu \mathrm{g} \mathrm{ml}^{-1}\right.$ in $50 \mathrm{mM}$ sodium carbonate buffer, $\mathrm{pH} 9.6$ ) overnight at $4{ }^{\circ} \mathrm{C}$. All subsequent washing steps between incubations were performed with PBST (PBS containing $0.05 \%, \mathrm{v} / \mathrm{v}$, Tween 20) (MacKenzie et al., 2009). Wells were blocked with $300 \mu$ l protein-free (PBS) blocking buffer (Thermo Scientific) for $2 \mathrm{~h}$ at room temperature. Volumes of anti-MUB antibody in dilutions ranging from $1: 900$ to $1: 218700$ in PBS $(100 \mu \mathrm{l})$ were then added to the coated wells and incubated for $2 \mathrm{~h}$ at room temperature. Pre-immune sera, in dilutions ranging from $1: 100$ to $1: 900$ in PBS, were included as negative controls. Antibody binding was detected after $1 \mathrm{~h}$ incubation with $100 \mu \mathrm{l}$ alkalinephosphatase-conjugated goat anti-rabbit $\operatorname{IgG}$ (whole molecule) $(1: 30000)$ at room temperature and $30 \mathrm{~min}$ incubation with $100 \mu \mathrm{l}$ p-nitrophenyl phosphate $\left(1 \mathrm{mg} \mathrm{ml}^{-1}\right)$ in $0.2 \mathrm{M}$ Tris, pH 9.6-10.5 (SIGMAFAST, Sigma) as substrate. The colorimetric assay was stopped with the addition of $3 \mathrm{M} \mathrm{NaOH}(100 \mu \mathrm{l}$ per well) and measured at $410 \mathrm{~nm}$ in an MRX II (Dynex) microtitre plate reader. All assays were performed in triplicate.

Immunodetection of MUB on the bacterial surface by ELISA. Bacteria were grown to stationary phase at $37{ }^{\circ} \mathrm{C}(20 \mathrm{~h})$, washed three times with PBS ( $\mathrm{pH} 7.4$ ) using 2 min centrifugation steps at $17000 \mathrm{~g}$, and resuspended with $\mathrm{PBS}$ to $\mathrm{OD}_{600}$ 0.5. Cells were transferred (100 $\mu \mathrm{l}$ per well) onto a high-binding microtitre plate (Greiner BioOne, Microlon 96); BSA ( $1 \mathrm{mg} \mathrm{ml}^{-1}$ in PBS) was included as negative control. Bacteria were incubated overnight at $4{ }^{\circ} \mathrm{C}$ and unbound cells were removed by washing with $300 \mu \mathrm{PBST}$ three times using a plate washer (Applied Quality Systems). Each well was blocked with $300 \mu \mathrm{l}$ protein-free (PBS) blocking buffer (Thermo Scientific) for $3 \mathrm{~h}$ at $4{ }^{\circ} \mathrm{C}$. After washing with PBST, $100 \mu \mathrm{l}$ anti-MUB antibody $(1: 2430$ in PBS) was added per well and incubated at room temperature for $2 \mathrm{~h}$. After further washing with PBST, alkaline-phosphatase-conjugated goat anti-rabbit IgG (whole molecule) was added and enzyme activity was measured as described above. All assays were performed in triplicate.

Microscopic immunolocalization of MUB on the bacterial surface. MUB was visualized on the surface of whole bacteria by immunogold electron microscopy. Bacteria were grown to early stationary phase in MRS broth $\left(20 \mathrm{~h}\right.$ incubation at $37^{\circ} \mathrm{C}$ ), washed once with PBS, as described above, and resuspended in PBS to $\mathrm{OD}_{600}$

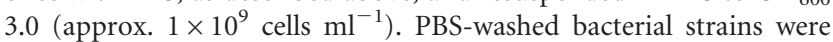
air-dried on to carbon-coated nickel grids and fixed by exposure to $25 \%(\mathrm{v} / \mathrm{v})$ glutaraldehyde vapour. Residual free aldehydes were blocked with PBS buffer ( $\mathrm{pH} 7.4$ ) containing $50 \mathrm{mM}$ glycine for $15 \mathrm{~min}$. The grids were incubated in goat blocking solution (Aurion) for $1 \mathrm{~h}$, washed three times for $5 \mathrm{~min}$ with incubation buffer (PBS containing $0.1 \%, \mathrm{w} / \mathrm{v}, \mathrm{BSA}-\mathrm{c}$; Aurion), then incubated overnight at $4{ }^{\circ} \mathrm{C}$ with anti-MUB polyclonal antibody $(1: 1000)$ in incubation buffer. Grids were washed six times for $10 \mathrm{~min}$ with incubation buffer and then incubated for $2 \mathrm{~h}$ at room temperature with goat anti-rabbit IgG conjugated with $15 \mathrm{~nm}$ gold particles (GAR-15, Aurion), diluted $1: 50$ in incubation buffer. Following six $5 \mathrm{~min}$ washes with incubation buffer and three 5 min washes with PBS, the bacteria were refixed in PBS containing $2 \%(\mathrm{v} / \mathrm{v})$ glutaraldehyde. After one 5 min wash with PBS and a final two 5 min washes with ultrapure water, the grids were examined and photographed in an FEI Tecnai
G2 20 Twin transmission electron microscope. Control grids which were treated only with the secondary antibody were included.

PCR amplification and DNA sequencing of mub and 16S rRNA gene fragments. Bacteria from mid- to late-exponential phase MRS cultures were harvested as described above and resuspended in sterile ultrapure water to $\mathrm{OD}_{600} 5.0$. PCRs $(50 \mu \mathrm{l})$ were carried out with $10 \mu \mathrm{l}$ of washed cells as template using HotStarTaq (Qiagen) and the gene-specific primers listed in Table 2 for 35 cycles. Amplified DNA fragments were purified by using PCR purification or gel extraction kits (Qiagen); their purity was confirmed by agarose gel electrophoresis and their concentration was measured in a NanoDrop ND-1000 spectrophotometer. DNA sequences were determined at the BBSRC Genome Analysis Centre (TGAC), Norwich, UK.

\section{RNA isolation and quantification of mub gene expression by} qRT-PCR. Total RNA was isolated from early- to mid-exponential phase bacterial cultures $\left(\mathrm{OD}_{600} \sim 0.6-1.0\right.$ in MRS) using the SV Total RNA Isolation System (Promega) and following a modified protocol for Gram-positive bacteria (N. Horn, IFR, personal communication). Briefly, the cell pellet from $1 \mathrm{ml}$ culture was washed twice with $1 \mathrm{ml}$ STE buffer (0.1 M NaCl, $10 \mathrm{mM}$ Tris/HCl, pH 8.0, $1 \mathrm{mM}$ EDTA) and, after centrifugation for $2 \mathrm{~min}$ at $14000 \mathrm{~g}$, the cells were resuspended in $100 \mu \mathrm{l}$ TE buffer containing $30 \mathrm{mg}$ hen egg white lysozyme $\mathrm{ml}^{-1}$ (93072 $\mathrm{U} \mathrm{mg}^{-1}$, Fluka). Cell wall digestion was carried out at $37{ }^{\circ} \mathrm{C}$ for $30 \mathrm{~min}$. The integrity and purity of the RNA preparation was determined by agarose gel electrophoresis and by spectrophotometry in a NanoDrop ND-1000. First-strand cDNA was synthesized using the QuantiTect reverse transcription kit (Qiagen) with random primers and qRT-PCR was carried out using the QuantiFast SYBR Green PCR kit in a 7300 Real-time PCR System (Applied Biosystems) with $m u b$ gene-specific primer pairs MucB1RIf/MucB1-RIr and MucB2-R4f/MucB2-R4r (Table 2). Signals were normalized to cDNA amplified from $16 \mathrm{~S}$ rDNA generated with primer pair 16S-341f/16S-534r (Table 2).

Determination of adherence to mucus. Mouse colonic mucus (MCM) was extracted from the large intestines of C57BL/6 wild-type mice. After washing with cold sterile PBS buffer ( $\mathrm{pH}$ 7.4) containing a complete protease inhibitor cocktail tablet (Roche), the mucosal surface was scraped with a glass slide to remove the mucus layer. Mucus scrapings were collected in ice-cold PBS $(1 \mathrm{ml})$ containing $4 \mathrm{M}$ guanidine hydrochloride (Sigma-Aldrich) and protease inhibitors. The tissue was homogenized with an Ultra-Turrax polytron (IKA) and a Status 70 ultrasonicator (Philip Harris Scientific) at $4{ }^{\circ} \mathrm{C}$, then centrifuged $\left(17000 \mathrm{~g}\right.$ for $1 \mathrm{~h}$ at $4{ }^{\circ} \mathrm{C}$ ) to remove cell debris. $L$. reuteri cells, grown in MRS overnight, were collected by centrifugation $\left(1342 \mathrm{~g}, 5 \mathrm{~min}, 15^{\circ} \mathrm{C}\right)$, washed twice with $\mathrm{PBS}$ and resuspended in PBS to $\mathrm{OD}_{600}$ 0.5. For the labelling of bacteria, cell suspensions were incubated with $10 \mu \mathrm{M}$ carboxyfluorescein diacetate (cFDA) (Sigma-Aldrich) at $37^{\circ} \mathrm{C}$ for $40 \mathrm{~min}$. Cell labelling and viability were checked by flow cytometry (FCM) analysis. Cells were then washed twice and resuspended in the same volume of PBS. MCM was diluted in PBS $\left(1 \mathrm{mg} \mathrm{ml}^{-1}\right)$ and $200 \mu \mathrm{l}$ was transferred into high-binding polystyrene microtitre plate wells (Greiner BioOne) and incubated overnight at $4{ }^{\circ} \mathrm{C}$. BSA $\left(1 \mathrm{mg} \mathrm{ml}^{-1}\right.$ in PBS $)$ was coated on to separate wells of the same plate as a negative control for adhesion. After washing three times with PBST $(200 \mu \mathrm{l})$ using a plate washer (Applied Quality Systems) to remove the excess mucus, $300 \mu \mathrm{l}$ protein-free blocking reagent (Thermo) was added per well and the plates were incubated at room temperature for $1 \mathrm{~h}$. After further washing, $200 \mu \mathrm{l}$ of the labelled bacterial suspension was added per well and the plates were incubated at $4{ }^{\circ} \mathrm{C}$ for $4 \mathrm{~h}$. After washing to remove unbound bacteria, bacteria bound to the mucus were lysed by incubation at $37{ }^{\circ} \mathrm{C}$ for $1 \mathrm{~h}$ with $1 \%(\mathrm{w} / \mathrm{v})$ SDS in $0.1 \mathrm{M} \mathrm{NaOH}$ $(200 \mu \mathrm{l})$. The released fluorescence was measured in a FLUOstar OPTIMA fluorometer (BMG Labtech) with excitation and emission 
Table 2. Oligonucleotide primers used

\begin{tabular}{|c|c|c|}
\hline Primer & Sequence $\left(3^{\prime}-5^{\prime}\right)$ & Gene (nt position)* \\
\hline $16 \mathrm{~S}-341 \mathrm{f}$ & CCTACGGGAGGCAGCAG & 16S rRNA (341-357) \\
\hline $16 \mathrm{~S}-534 \mathrm{r}$ & ATTACCGCGGCTGCTGG & 16S rRNA (518-534) \\
\hline $16 \mathrm{~S}-1577 \mathrm{r}$ & AAGGAGGTGATCCAGCCGCA & 16S rRNA (1558-1577) \\
\hline MucB1-RIf & ATGCAAGAAGCTGCCATCAG & Mub-RI (1789-1805) and Mub-RIII (2959-2975)† \\
\hline MucB1-RIr & TTATGCCTTTGAGCTAGTTG & Mub-RI (2039-2055) \\
\hline MucB1-RVIf & ATGCAAGAAGCTCAAGCCATC & Mub-RVI (9064-9081) \\
\hline MucB2-RVIr & TTAATCAAGCTTCTTGTAGGT & Mub-RVI (9664-9681) \\
\hline MucB2-R4f & ATGGGTACGAAGACGCTGAC & $\begin{array}{l}\text { Mub-R2 (4921-4937), Mub-R4 (6025-6041), Mub-R5 (6577-6593), } \\
\text { Mub-R6 (7129-7145), Mub-R7 (7681-7697), Mub-R8 (8233-8249) }\end{array}$ \\
\hline MucB2-R4r & TTAGGCATCAGCCGTGTAGA & $\begin{array}{l}\text { Mub-R2 (5186-5202), Mub-R4 (6290-6306), Mub-R5 (6842-6858), } \\
\text { Mub-R6 (7394-7410), Mub-R7 (7946-7962), Mub-R8 (8498-8514) }\end{array}$ \\
\hline
\end{tabular}

${ }^{\star}$ Nucleotide positions are given relative to the complete 16S rRNA (NC_009513; Lreu_R0003) and mub (AF120104) genes.

$\dagger$ Includes a 1 bp mismatch at the $3^{\prime}$ end.

$\$$ Primer 1063Mub-3'r anneals in the 3' region downstream from the published mub genomic sequence (AF120104) and is based on the draft genome sequence of strain ATCC 53608 (unpublished data).

wavelengths of $485 \mathrm{~nm}$ and $520 \mathrm{~nm}$, respectively. Adhesion was calculated as the percentage of fluorescence recovered after binding relative to the fluorescence of the bacterial suspension added to the mucus or BSA and the values were normalized to the percentage adhesion values of ATCC 53608, which was used as an internal control on each plate. Each assay was performed in triplicate for each strain and on at least three separate occasions. The effect of antisera on bacterial adhesion to the mucus was studied by pre-incubating the labelled bacterial suspension with anti-MUB antibody, or preimmune serum as a control, at appropriate concentrations $(0-1 \%$, $\mathrm{v} / \mathrm{v}$, antibody/serum) at room temperature for $1 \mathrm{~h}$ prior to addition to the mucus-coated wells.

Characterization of cell-surface extracts and spent media by gel electrophoresis, Western blotting and mass spectrometry. Bacteria were cultured in $20 \mathrm{ml}$ MRS broth until early stationary phase and harvested by centrifugation at $1342 \mathrm{~g}$ for $10 \mathrm{~min}$. Spent, centrifuged growth medium in either $2 \mathrm{ml}$ or $10 \mathrm{ml}$ volumes was retained for concentration using Vivaspin-2 or Vivaspin-6 ultrafiltration $100 \mathrm{kDa}$ molecular weight (mass) cut-off (MWCO) spin columns, respectively (Sartorius Stedim Biotech), at $4{ }^{\circ} \mathrm{C}$, following the manufacturer's instructions. The concentrated spent media samples were then buffer-exchanged with PBS $(1 \mathrm{ml})$ and centrifuged to give a final concentration approximately $50 \times$ to $70 \times$. Cell-surface extracts were prepared from PBS-washed bacteria following the method described by Kankainen et al. (2009) with modifications. Briefly, cells were disrupted by vortexing with an equal volume of glass beads (425-600 $\mu \mathrm{m}$ diameter, Sigma) and $17 \mathrm{ml}$ PBS per g wet weight of cells for three 2 min pulses at full speed with 2 min intervals on ice between pulses. Samples of cell-surface extracts, soluble cytoplasmic extracts and concentrated spent media were analysed by SDS-PAGE using NuPAGE 4-12\% Bistris gels (Invitrogen) with MOPS SDS running buffer ( $50 \mathrm{~min}$ at $200 \mathrm{~V}$ constant voltage) and HiMark Unstained High Molecular Weight Protein Standard (Invitrogen) as a marker. Gels were stained with the Colloidal Blue staining kit (Invitrogen) and scanned in a GS-800 calibrated densitometer (Bio-Rad). When required, proteins were electroblotted onto an Immobilon-P membrane $(0.45 \mu \mathrm{m}$ pore size, Millipore) for Western blot detection with anti-MUB polyclonal antibody (1:1000), alkaline-phosphatase-conjugated goat anti-rabbit IgG (whole molecule) $(1: 30000)$ and $100 \mu \mathrm{g}$ nitro blue tetrazolium $\mathrm{ml}^{-1}, 50 \mu \mathrm{g} \mathrm{5-}$ bromo-4-chloro-3-indolyl phosphate-toluidine $\mathrm{ml}^{-1}$ in $4 \mathrm{mM} \mathrm{MgCl}_{2}$ and $100 \mathrm{mM}$ Tris/ $\mathrm{HCl}$ (pH 9.6) as substrate. Trypsinized samples of proteins excised from SDS-PAGE gels were analysed in an Ultraflex MALDI-TOF/TOF mass spectrometer (Bruker Daltonics) and the MS data were used to search against the relevant sequence databases as described previously (MacKenzie et al., 2009) using the Mascot 2.2.06 search engine (Matrix Science).

Cell aggregation and cell-surface MUB quantification by FCM. Bacterial cultures were grown in triplicate to early stationary phase [20 h growth in $20 \mathrm{ml}$ filtered $(0.2 \mu \mathrm{m})$ MRS broth at $37^{\circ} \mathrm{C}$ ]. Aliquots $(1 \mathrm{ml})$ were harvested and washed once with filtered $(0.2 \mu \mathrm{m})$ PBS buffer, which was also used for all subsequent washing and resuspension steps. The cell suspensions were adjusted to $\mathrm{OD}_{600}$ $\sim 2.5$ in PBS.

Cell aggregation of PBS-washed cells was quantified by measuring forward scatter (FSC) and side scatter (SSC) signals detected by photodiode and photomultiplier tube (PMT) sensors, respectively. In addition, cell aggregation of unwashed cells was determined using a more gentle approach by minimizing the number of sample-handling steps whereby $5 \mu \mathrm{l}$ of unwashed MRS culture was gently mixed with $1 \mathrm{ml}$ PBS. During data analysis, a gate for events with high FSC and high SSC was used to determine the percentage of aggregating cells.

To determine the production of MUB, aliquots $(50 \mu \mathrm{l})$ of PBSwashed cells were incubated with polyclonal anti-MUB antibody $(1: 100)$ in the dark, on ice for $30 \mathrm{~min}$. Following a wash with $1 \mathrm{ml}$ PBS, the cells were resuspended in $25 \mu$ PBS containing fluoresceinconjugated goat anti-rabbit IgG (whole molecule) antibody $(1: 26)$ in the dark, at room temperature for $15 \mathrm{~min}$. Subsequently, $20 \mu \mathrm{l}$ of cell suspension was diluted in $1 \mathrm{ml} \mathrm{PBS}, 1 \mu \mathrm{l}$ propidium iodide (PI, $1.0 \mathrm{mg} \mathrm{ml}{ }^{-1}$, Invitrogen Molecular Probes) was added and the sample was analysed in a FACSCalibur flow cytometer (Becton 
Dickinson) using CellQuestPro software. A total of at least 20000 events per sample were acquired at low flow rate. PMT sensors in channels FL1 $(530 / 30 \mathrm{~nm})$ and FL2 $(585 / 42 \mathrm{~nm})$ were used to quantify green fluorescein and orange PI fluorescence, respectively. FCM data were analysed using FlowJo (TreeStar).

\section{RESULTS AND DISCUSSION}

\section{Binding of $L$. reuteri strains to mucus in vitro is strain-specific}

The adhesion values of the different $L$. reuteri strains tested are reported in Fig. 1. All the strains tested display an identity of at least $99.5 \%$ to the $16 \mathrm{~S}$ rRNA sequence of the type strain (DSM 20016 ${ }^{\mathrm{T}}$ ) (Oh et al., 2010). The L. reuteri strains varied markedly in their ability to bind to mouse mucus in vitro, with substantial differences reflected in percentages of adhesion, ranging from $0.16 \%( \pm 0.07 \%)$ to $34.66 \%( \pm 0.52 \%)$. L. reuteri ATCC 53608 and ATCC 55739 showed the highest binding to mouse mucus (Fig. 1); both strains were also shown to adhere most efficiently to pig gastric mucin-coated wells (not shown). In contrast, strains MF14-C, MF2-3 and LB54 showed a lower percentage of adhesion to mucus than to BSA, with only the latter two being significantly different $(P<0.05)$ (Fig.
1). The adhesion values of strains $1063 \mathrm{~N}, \mathrm{Lp} 167-67,20-2$, 3c6, \#20, r13, Lr4020, ML1, CR, One-One, DSM 17509, N2D and R2LC to mucus-coated wells were similar to those detected in BSA-coated wells $(<0.8 \%$; Fig. 1$)$. These results agree with previous reports showing that the binding properties of lactobacilli to host intestinal mucus in vitro are strain-specific, although those studies were limited to a much smaller number of strains (Ouwehand et al., 2001; Uchida et al., 2004; Vesterlund et al., 2006; Collado et al., 2007; Kinoshita et al., 2007; Li et al., 2008; Tallon et al., 2007; Muñoz-Provencio et al., 2009).

Host-specific adherence is regarded as a desirable property for probiotic bacteria and is recommended as one of the selection criteria. A remarkable feature of the $L$. reuteri population is how it has diversified to form ecotypes that are highly host-specific (Oh et al., 2010). However, our in vitro binding assay showed no clear correlation between the host of origin of the bacteria and the origin of the intestinal mucus (Fig. 1). For example, strains ATCC 53608 and ATCC 55739, originating from pig and rat, respectively, adhered most efficiently to mouse colonic mucus (Fig. 1) and adhered without discrimination to pig gastric mucin (data not shown). This lack of host specificity in relation to mucus adhesion in vitro had been reported for $L$. reuteri

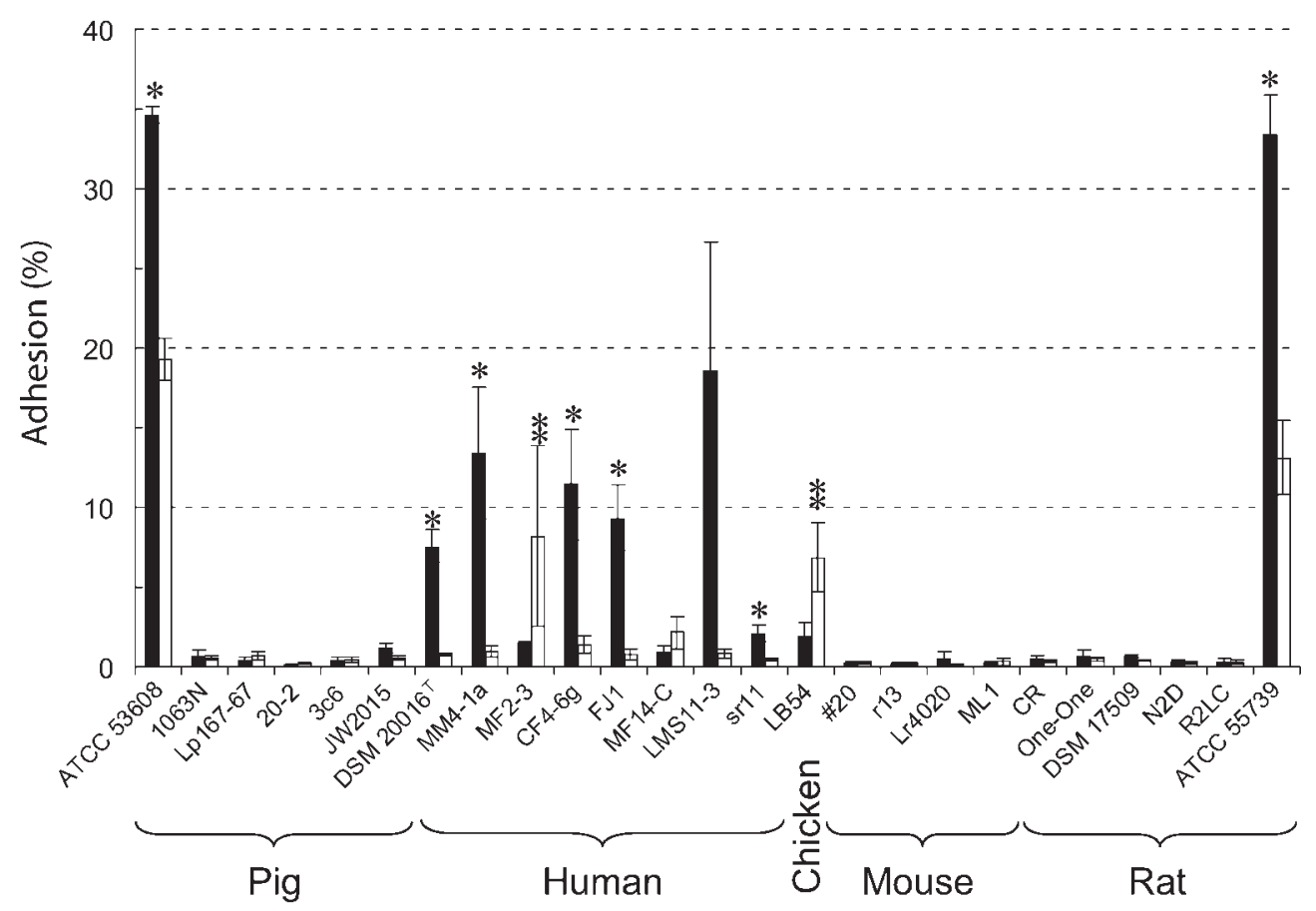

Fig. 1. Adhesion of $L$. reuteri strains to MCM (black bars) and BSA (white bars). L. reuteri strains are grouped in relation to the host from which they were isolated. Microtitre plate adhesion assays were carried out in triplicate for each strain and on at least three separate occasions as described in Methods. Results from different plates are expressed as percentage binding values normalized to the mean value for strain ATCC 53608, used as an internal control on each plate. SD $(n \geqslant 3)$ is indicated by error bars. Strains where binding to MCM was significantly higher than to BSA, as determined by the Student's $t$-test $(P<0.05)$, are indicated by a single asterisk, while strains showing significantly higher binding to BSA than to MCM are indicated with a double asterisk. 
strain ATCC 53608, which showed binding to pig but also to hen small intestinal mucus (Roos \& Jonsson, 2002). Similarly, the binding properties of probiotic lactic acid bacteria to host intestinal mucus were considered to be more dependent on the strain than on the host (Rinkinen et al., 2003). In contrast, in vivo competitive colonization using gnotobiotic mouse models revealed that most rodent L. reuteri isolates, including strain R2LC from a rat, showed elevated ecological performance in the murine gut (Schreiber et al., 2009; Oh et al., 2010). This absence of correlation between in vitro adhesion assays and in vivo colonization studies highlights the multifactorial nature of the colonization process and the need for caution when using mucus adhesion as a measure of colonization performance.

In our assay, adhesion is a reflection of both the mucus recognition abilities and the aggregation behaviour of the bacterial strains. The apparent mucus-binding property reported here for strains ATCC 53608 and ATCC 55739 could be related to the ability of these strains to form multicellular aggregates. Indeed, FCM revealed that the aggregation levels of PBS-washed cells of $L$. reuteri ATCC 53608 and ATCC 55739 were significantly higher than those of strains $1063 \mathrm{~N}$ and DSM $20016^{\mathrm{T}}$ (Fig. 2). It should be noted here that strain DSM 20016 ${ }^{\mathrm{T}}$ shows high aggregation during growth in MRS broth but, even when handled gently, this strain displayed only about $4 \%$ cell aggregates when diluted directly into PBS (data not shown), indicating that aggregation is a mulifactorial process affected by ionic strength and/or $\mathrm{pH}$, and is strain-dependent. High levels of autoaggregation of PBSwashed cells have previously been reported in some $L$. reuteri strains, and a protein $(\mathrm{AggH})$ from strain ATCC 53608 with homology to ATP-dependent RNA helicases was identified as one of the effector molecules mediating aggregation (Roos et al., 1999). Similarly, exopolysaccharides (EPS), which contribute to cell-surface physicochemical properties, have been implicated in the L. reuteri aggregation phenotype, as supported by the implication of EPS-producing enzymes (GtfA and Inu) in cell aggregation and biofilm formation (Walter et al., 2008).

\section{The expression and production of MUB in $L$. reuteri is strain-dependent}

The L. reuteri MUB and MUB-like sequences have characteristics typical of cell-surface proteins of Grampositive bacteria: an $\mathrm{N}$-terminal signal peptide (YSIRK motif), targeting the protein for secretion, and a Cterminal sortase recognition site (LPxTG), targeting the protein for covalent attachment to the peptidoglycan layer on the outside of the bacterial cell (Ton-That et al., 2004). The diversity of MUBs among $L$. reuteri strains was first explored at the protein level using immunodetection assays. Of the 25 strains screened by ELISA, MUB was detected on the cell surface of strains ATCC 53608 and ATCC 55739 (Fig. 3). FCM was used to further quantify the level of MUB present on the surface of live and dead

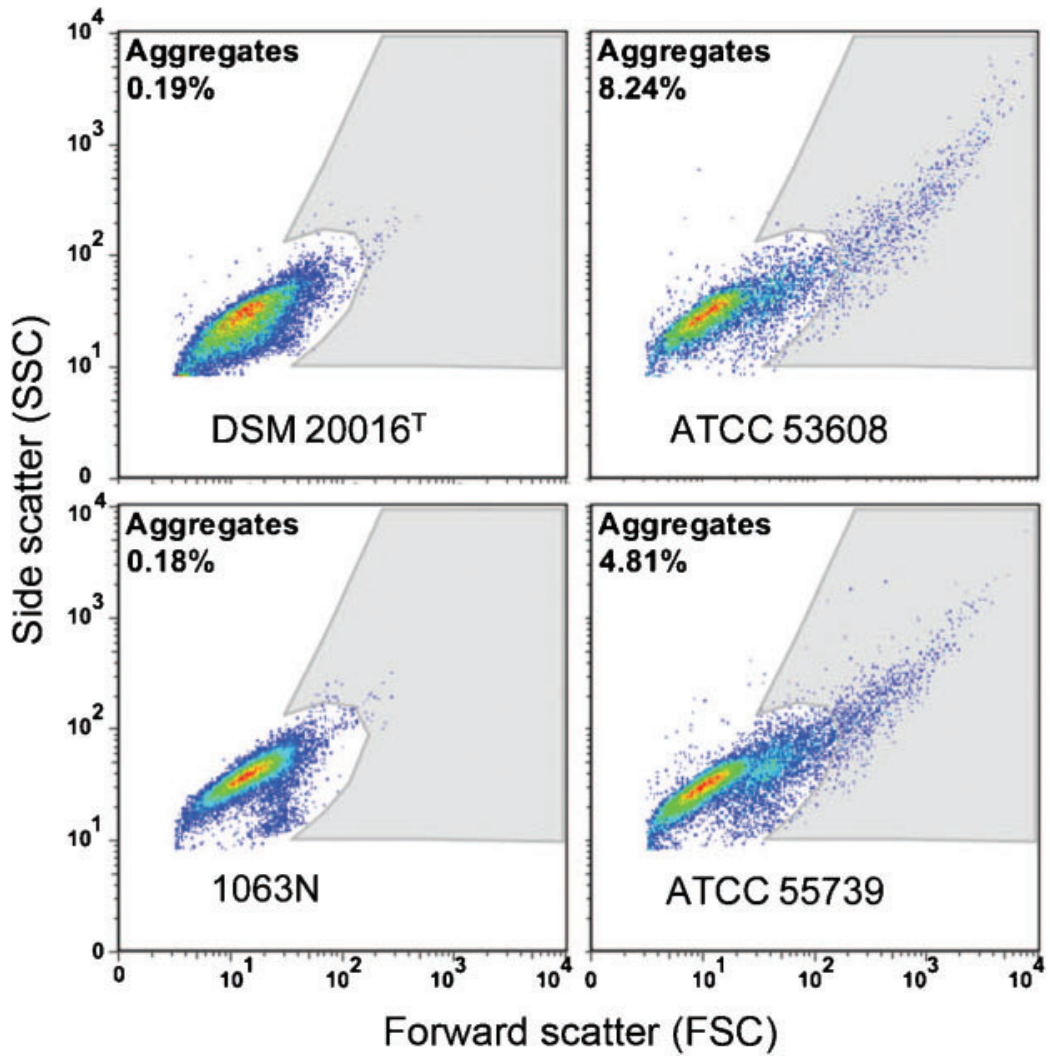

Fig. 2. Autoaggregation of $L$. reuteri strains. PBS-washed bacterial cells were analysed using FCM and cell aggregation was quantified by determining the percentage of events with a high SSC and FSC. These cell clumps or aggregates consist of multiple cells and can be homogenized into a single cell suspension using sonication (data not shown). 


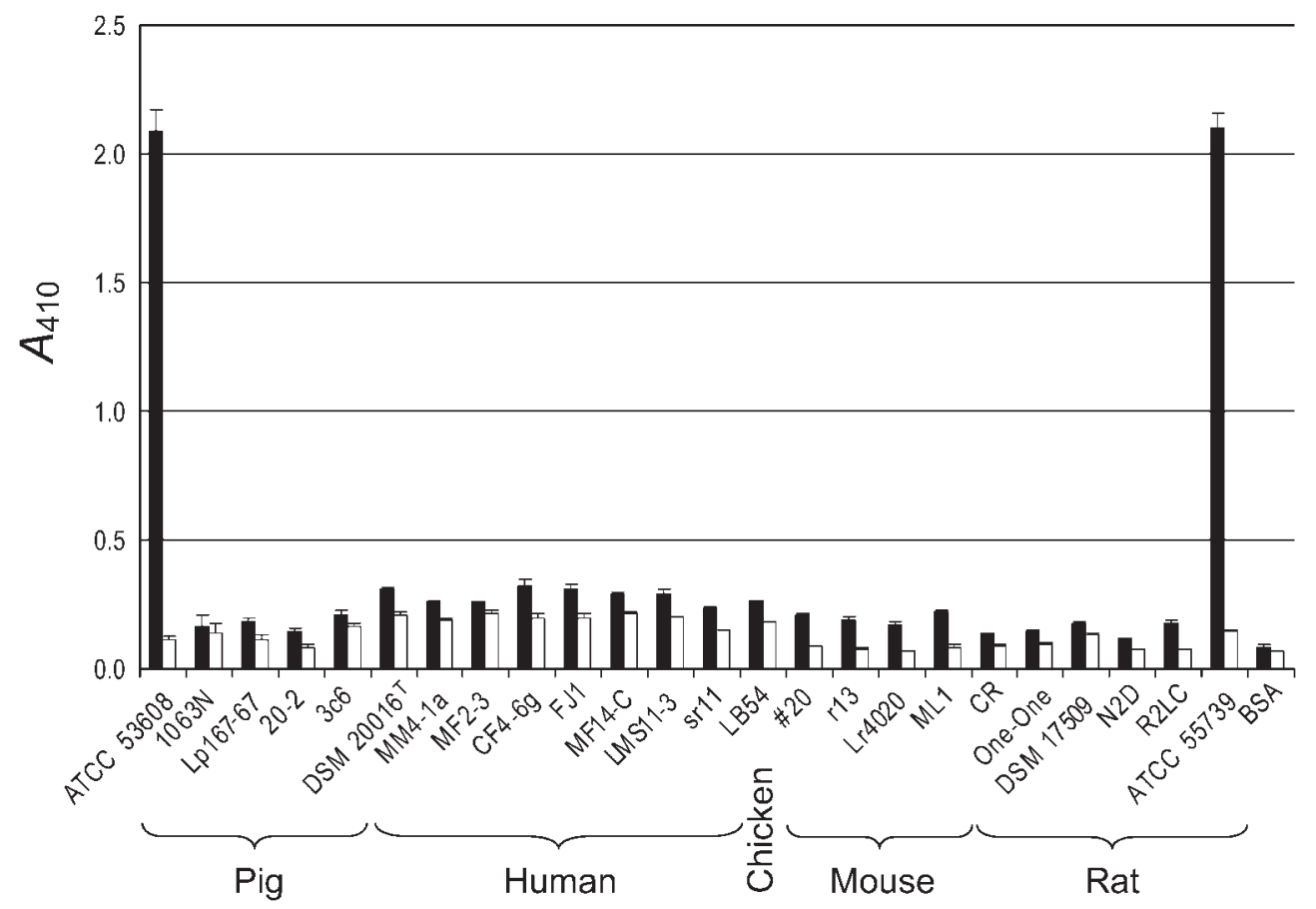

Fig. 3. Immunodetection of MUBs by ELISA. L. reuteri strains are grouped according to the host from which they were isolated. Bacteria were allowed to adhere to the surface of microtitre plates and were probed with polyclonal anti-MUB antibody and fluorescein-conjugated secondary antibody (black bars) or with the secondary antibody only (white bars). Negative controls with BSA-coated wells were also included. SD is indicated by error bars; for all strains $n=3$, except for ATCC $53608(n=9)$ and DSM $20016^{\top}(n=12)$.

cells in a subset of selected $L$. reuteri strains with varying mucus-adhesion capacities and isolated from a variety of hosts: strains ATCC 53608 (pig), ATCC 55739 (rat), 1063N (pig), and the type strain DSM 20016 ${ }^{\mathrm{T}}$ (human) (Supplementary Fig. S1, available with the online version of this paper). The results were in agreement with the ELISA data, with strains ATCC 53608 and ATCC 55739 showing the highest MUB levels and having a significant 150-200-fold difference in fluorescence intensity for live and dead cells compared to strains $1063 \mathrm{~N}$ and DSM $20016^{\mathrm{T}}$. Surprisingly, although strains $1063 \mathrm{~N}$ and ATCC 53608 originate from the same porcine isolate (Roos \& Jonsson, 2002; Wadström et al., 1987) and the mub gene could be detected by PCR in both strains, no MUB was detected on the surface of $1063 \mathrm{~N}$.

To further characterize the postulated relationship between variation in MUB production and mucus adhesion and/or aggregation capacity, the diversity of mub genes among $L$. reuteri strains was further explored by PCR amplification and DNA sequencing. The presence of the mub gene was confirmed in strains ATCC 53608 and ATCC 55739, both of which produce MUB, showing $100 \%$ identity to the published sequence in GenBank (AF120104), while no mub gene was amplified from DSM $20016^{\mathrm{T}}$, as was expected from its genome sequence (NCBI reference sequence: NC_009513.1). Surprisingly, strain $1063 \mathrm{~N}$ was also shown to contain the mub gene. qRT-PCR was therefore carried out to determine whether the lack of MUB expression was occurring at the transcriptional level in this strain. As shown in Supplementary Table S1, the level of $m u b$ transcripts produced by strain $1063 \mathrm{~N}$ was comparable to that of ATCC 53608, when expressed relative to that of $16 \mathrm{~S}$ rRNA.

To confirm these results at the protein level, the production of MUB and other high-molecular-mass cell-wall proteins was investigated by analysing spent culture medium and cell-wall extracts from strains ATCC 53608, ATCC 55739, $1063 \mathrm{~N}$ and DSM $20016^{\mathrm{T}}$ by gel electrophoresis (Fig. 4a). As reported previously by Roos \& Jonsson (2002), and confirmed here, a substantial amount of MUB was released into the growth medium by strain ATCC 53608. An apparent lower level of MUB was produced by strain ATCC 55739, in both the medium and the cell wall extract, in agreement with the FCM data. The production of a highmolecular-mass protein by strain DSM $20016^{\mathrm{T}}$ was also observed in the growth medium and identified by MALDITOF-MS as the cell-wall LPxTG protein LAR_0958 from DSM $20016^{\mathrm{T}}$. Although not classified as a MucBP protein, this protein has similarities with members of this protein family and could possibly be involved in the relatively high mucus binding detected in strain DSM 20016 (Fig. 1).

Interestingly, strain $1063 \mathrm{~N}$ did not produce a protein of the same size as full-length MUB but released a substantial 
(a)

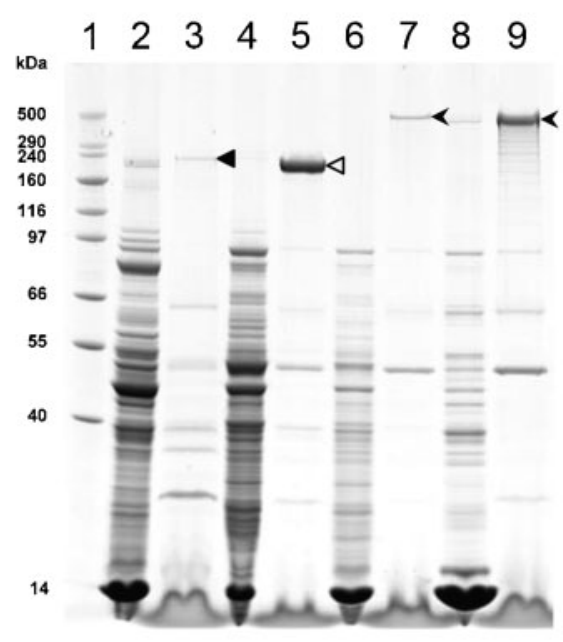

(b)

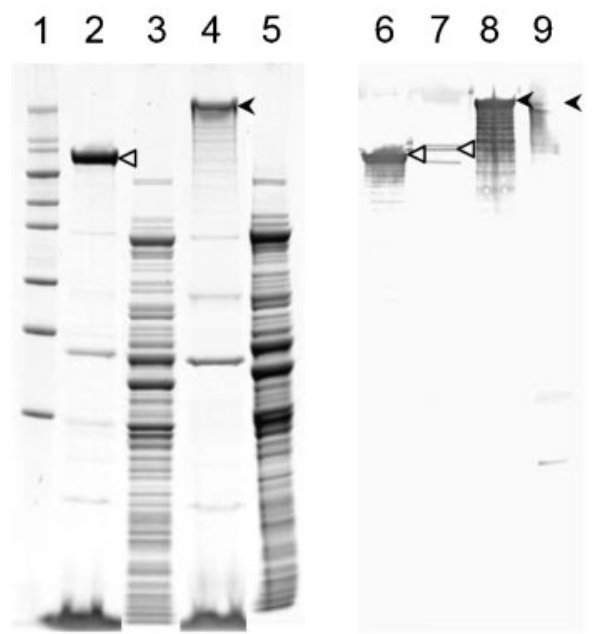

Fig. 4. (a) SDS-PAGE of cell wall extracts (CWE) and spent medium (SM) samples from L. reuteri strains. Lane 1, HiMark MW marker; lane 2, DSM $20016^{\top}$ CWE; lane 3, DSM $2006^{\top}$ SM; lane 4, 1063N CWE; lane 5, 1063N SM; lane 6, ATCC 55739 CWE; lane 7, ATCC 55739 SM; lane 8, ATCC 53608 CWE; lane 9, ATCC 53608 SM. The ATCC 53608 and ATCC 55739 MUBs are indicated by the arrowheads, the truncated MUB from $1063 \mathrm{~N}$ by the open triangle and the DSM $20016^{\top}$ lar_0958 protein by the solid triangle. (b) SDS-PAGE and anti-MUB Western blot of spent medium (SM) and soluble cytoplasmic extracts (SCE) from L. reuteri strains 1063N and ATCC 53608. Lane 1, HiMark MW marker; lanes 2 and 6, 1063N SM; lanes 3 and 7, 1063N SCE; lanes 4 and 8, ATCC 53608 SM; lanes 5 and 9, ATCC 53608 SCE. MUBs are indicated using the same symbols as in (a).

amount of a protein with a size of approximately $200 \mathrm{kDa}$ into the medium (Fig. 4a). This shortened version of MUB still reacted with the anti-MUB polyclonal antiserum and weak signals could also be seen in the soluble cytoplasmic extract from strain $1063 \mathrm{~N}$, perhaps due to protein that was transiently present during translation, prior to secretion through the cell wall (Fig. 4b). After DNA sequencing of the PCR fragment amplified from strain 1063N using primers 1063Mub-R1f/1063Mub-R24r, the MUB truncation was shown to be due to a frameshift caused by the insertion of a direct repeat sequence of $13 \mathrm{bp}\left(5^{\prime}\right.$ CACGGTAATCTTC-3') at position $4867 \mathrm{nt}$ in the wildtype mub gene (Supplementary Table S2). This frameshift created a premature stop codon that resulted in a truncated mature protein (after removal of the putative N-terminal 49 aa secretion signal) of 1541 aa with an estimated mass of $169 \mathrm{kDa}$, which is close to the apparent mass of $\sim 200 \mathrm{kDa}$ observed in SDS-PAGE and predicted from MALDI-TOF-MS data. The equivalent PCR fragment amplified from ATCC 53608 lacked the 13 bp insertion as expected. However, the $3^{\prime}$-terminal $1 \mathrm{~kb}$ of the $m u b$ gene from strain 1063N, including the Mub-RVI- and LPxTGencoding regions, was $100 \%$ identical at the DNA level to the wild-type mub gene from ATCC 53608 (Supplementary Table S2). This is consistent with the mutant mub gene (and cDNA) in strain $1063 \mathrm{~N}$ being full-length, but its mRNA being translated to give a truncated MUB that only contains Mub repeats RI, RII, RIII, RIV, R1 and part of R2, compared with the $14 \mathrm{Mub}$ repeats in the full-length MUB, which is in excellent agreement with MALDI-TOF-MS results. The truncated protein also lacks the LPxTG cellwall anchor motif and thus the ability to attach to the cell surface, which is in agreement with the ELISA and FCM data. This spontaneous mutation may be the result of an adaptive response to the in vitro environment as is postulated for lactobacilli and other bacteria (Båth et al., 2005). Indeed, a putative, highly truncated YSIRK-containing protein ( $\mathrm{Lr} 1997)$ with $89 \%$ similarity to the $\mathrm{N}$-terminal 39 aa of the 12 MucBP repeat protein Lreu23DRAFT_4946 from $L$. reuteri strain 100-23 was identified on the pLR581 plasmid of $L$. reuteri ATCC 55730, although the gene product was not characterized further (Båth et al., 2005; Rosander et al., 2008). We propose to designate the mutated mub gene in strain $1063 \mathrm{~N}$ as $m u b-4867^{\mathrm{i}}$. The location of MUB on the bacterial cell surface of ATCC 53608 and ATCC 55739 strains was confirmed by immunogold electron microscopy, while negligible or no MUB could be detected in the cell walls of strains 1063N and DSM $20016^{\mathrm{T}}$, respectively (Supplementary Fig. S2).

\section{MUB contributes to the in vitro mucus-binding and aggregation properties of $L$. reuteri strains}

From the results presented above, the mucus-binding properties of the $L$. reuteri strains tested in this study appear to correlate with the presence of MUB at the bacterial cell surface. Individual recombinant Mub repeats have been shown to adhere to mucus (Roos \& Jonsson, 


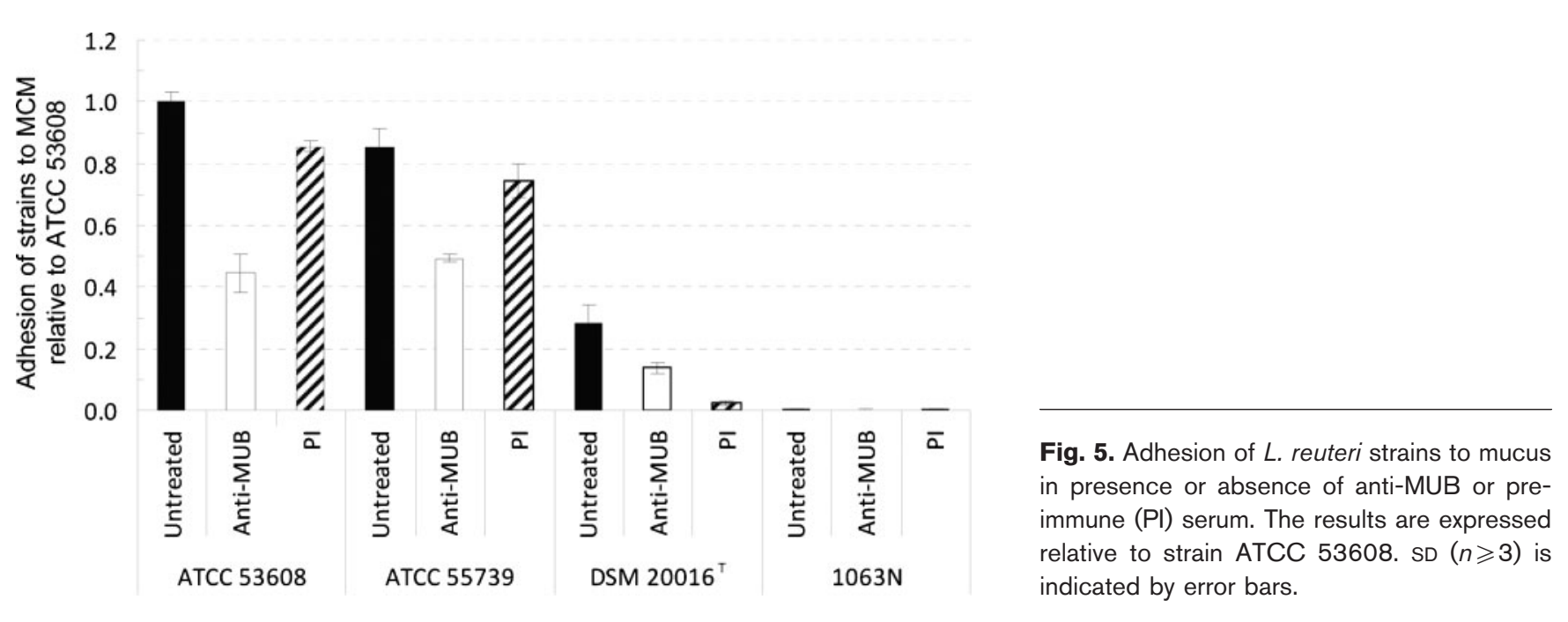

2002, and unpublished data). To further characterize the contribution of MUB to the interaction of the whole bacteria with mucus, a polyclonal antibody that recognized Mub repeats was tested for inhibition of the interaction using strains ATCC 53608, ATCC 55739, $1063 \mathrm{~N}$ and DSM $20016^{\mathrm{T}}$. The antibodies were shown to reduce the binding of strains ATCC 53608 and ATCC 55739 to mucus by $55.4 \%$ and $42.3 \%$, respectively, while IgG from pre-immune serum had no effect on the binding of the bacteria, showing that MUB contributes to the binding of the bacteria to mucus (Fig. 5). Strain 1063N, which did not exhibit a significant mucus-binding ability, remained unaffected by the antibody treatment, whereas the binding of strain DSM 20016 ${ }^{\mathrm{T}}$ to mucus was completely inhibited by the pre-immune serum treatment, lending evidence to the multifactorial nature of mucus adhesion. The contribution of MUB to bacterial adherence to mucus can be explained by its strain-specific role in the recognition of mucus elements and/or in its ability to promote aggregation. It has been suggested that the majority of the aggregation interactions between gut isolates are mediated by lectin-carbohydrate interactions (Ledder et al., 2008). Although a direct interaction of MUB with specific glycans remains to be demonstrated, competitive adhesion assays showed that such an interaction can be significantly reduced by the addition of specific sugars (Bumbaca et al., 2007; Pretzer et al., 2005; Roos \& Jonsson, 2002), suggesting a lectin-type mediated interaction and a potential role for MUB in cell aggregation.

\section{Concluding remarks}

In conclusion, our study has shown that MUB contributes to the strain-specific ability of $L$. reuteri to bind to mucus and autoaggregate in vitro, although the mechanisms need to be investigated further. This property is considered to provide an ecological advantage for extended persistence in the gut and to ensure the optimal functionality and expression of the health-promoting physiological functions of probiotics (Kleerebezem \& Vaughan, 2009). The spontaneous (non-GM) mutant expressing truncated MUB characterized here will serve as a valuable tool to assess the impact of MUB on gut colonization in vivo. Interestingly, the MUB under study was only detectable in L. reuteri strains ATCC 53608, ATCC 55739 and $1063 \mathrm{~N}$ (albeit in truncated form in the latter). These strains are indistinguishable by amplified fragment length polymorphism and multi-locus sequence typing analysis (Oh et al., 2010), and the mub gene sequences are also $100 \%$ identical, except for the $13 \mathrm{bp}$ insertion in $m u b-4867^{\mathrm{i}}$ (Supplementary Table S2). Therefore, this study has revealed that the particular MUB investigated is highly specific to a very small set of closely related strains of $L$. reuteri. This is despite the fact that 17 proteins with a putative MucBP domain can be found in the available genomes of $L$. reuteri strains 100-23, DSM $20016^{\mathrm{T}}$, MM2-3, MM4-1, ATCC 55730 and CF48-3A, nine of which were present in the rodent isolate 100-23. However, all these proteins show $<25 \%$ homology to the ATCC 53608 MUB, and several of the MUB in L. reuteri strains DSM $20016^{\mathrm{T}}$, MM2-3, MM4-1 are probably truncated through pseudogene formation. Therefore, it appears that although proteins with mucus-binding domains are important in the biology of $L$. reuteri or played important roles during the evolution of the species, they show a remarkable degree of variability. The ecological and evolutionary forces that cause this variation remain an important area for future research.

\section{ACKNOWLEDGEMENTS}

The authors would like to thank Fran Mulholland (IFR) for MALDI-TOF-MS protein analysis, Phaik Lyn Oh (University of Nebraska) for supplying L. reuteri strains and advising on culture conditions, and Christine Fuell, Maddy Houchen, Ola Symes and Kathryn Cross (IFR) for excellent technical support. This work was supported by the Biotechnology and Biological Sciences Research Council (BBSRC). 


\section{REFERENCES}

Abbas Hilmi, H. T., Surakka, A., Apajalahti, J. \& Saris, P. E. (2007). Identification of the most abundant Lactobacillus species in the crop of 1- and 5-week-old broiler chickens. Appl Environ Microbiol 73, 7867-7873.

Båth, K., Roos, S., Wall, T. \& Jonsson, H. (2005). The cell surface of Lactobacillus reuteri ATCC 55730 highlighted by identification of 126 extracellular proteins from the genome sequence. FEMS Microbiol Lett 253, 75-82.

Boekhorst, J., Helmer, Q., Kleerebezem, M. \& Siezen, R. J. (2006). Comparative analysis of proteins with a mucus-binding domain found exclusively in lactic acid bacteria. Microbiology 152, 273-280.

Brooks, S. P., McAllister, M., Sandoz, M. \& Kalmokoff, M. L. (2003). Culture-independent phylogenetic analysis of the faecal flora of the rat. Can J Microbiol 49, 589-601.

Bumbaca, D., Littlejohn, J. E., Nayakanti, H., Lucas, A. H., Rigden, D. J., Galperin, M. Y. \& Jedrzejas, M. J. (2007). Genome-based identification and characterization of a putative mucin-binding protein from the surface of Streptococcus pneumoniae. Proteins 66, 547-558.

Collado, M. C., Grześkowiak, k. \& Salminen, S. (2007). Probiotic strains and their combination inhibit in vitro adhesion of pathogens to pig intestinal mucosa. Curr Microbiol 55, 260-265.

Connolly, E. (2009). State of the art on research of Lactobacillus reuteri. Minerva Pediatr 61, 634-636.

Dethlefsen, L., Huse, S., Sogin, M. L. \& Relman, D. A. (2008). The pervasive effects of an antibiotic on the human gut microbiota, as revealed by deep 16S rRNA sequencing. PLoS Biol 6, e280.

Heilig, H. G., Zoetendal, E. G., Vaughan, E. E., Marteau, P., Akkermans, A. D. \& de Vos, W. M. (2002). Molecular diversity of Lactobacillus spp. and other lactic acid bacteria in the human intestine as determined by specific amplification of $16 \mathrm{~S}$ ribosomal DNA. Appl Environ Microbiol 68, 114-123.

Kankainen, M., Paulin, L., Tynkkynen, S., von Ossowski, I., Reunanen, J., Partanen, P., Satokari, R., Vesterlund, S., Hendrickx, A. P. A. \& other authors (2009). Comparative genomic analysis of Lactobacillus rhamnosus GG reveals pili containing a human-mucus binding protein. Proc Natl Acad Sci U S A 106, 17193-17198.

Kinoshita, H., Uchida, H., Kawai, Y., Kitazawa, H., Miura, K., Shiiba, K., Horii, A. \& Saito, T. (2007). Quantitative evaluation of adhesion of lactobacilli isolated from human intestinal tissues to human colonic mucin using surface plasmon resonance (BIACORE assay). J Appl Microbiol 102, 116-123.

Kleerebezem, M. \& Vaughan, E. E. (2009). Probiotic and gut lactobacilli and bifidobacteria: molecular approaches to study diversity and activity. Annu Rev Microbiol 63, 269-290.

Kleerebezem, M., Hols, P., Bernard, E., Rolain, T., Zhou, M., Siezen, R. J. \& Bron, P. A. (2010). The extracellular biology of the lactobacilli. FEMS Microbiol Rev 34, 199-230.

Ledder, R. G., Timperley, A. S., Friswell, M. K., Macfarlane, S. \& McBain, A. J. (2008). Coaggregation between and among human intestinal and oral bacteria. FEMS Microbiol Ecol 66, 630-636.

Leser, T. D., Amenuvor, J. Z., Jensen, T. K., Lindecrona, R. H., Boye, M. \& Møller, K. (2002). Culture-independent analysis of gut bacteria: the pig gastrointestinal tract microbiota revisited. Appl Environ Microbiol 68, 673-690.

Ley, R. E., Hamady, M., Lozupone, C., Turnbaugh, P. J., Ramey, R. R., Bircher, J. S., Schlegel, M. L., Tucker, T. A., Schrenzel, M. D. \& other authors (2008). Evolution of mammals and their gut microbes. Science 320, 1647-1651.
Li, X. J., Yue, L. Y., Guan, X. F. \& Qiao, S. Y. (2008). The adhesion of putative probiotic lactobacilli to cultured epithelial cells and porcine intestinal mucus. J Appl Microbiol 104, 1082-1091.

MacKenzie, D. A., Tailford, L. E., Hemmings, A. M. \& Juge, N. (2009). Crystal structure of a mucus-binding protein repeat reveals an unexpected functional immunoglobulin binding activity. J Biol Chem 284, 32444-32453.

Muñoz-Provencio, D., Llopis, M., Antolín, M., de Torres, I., Guarner, F., Pérez-Martínez, G. \& Monedero, V. (2009). Adhesion properties of Lactobacillus casei strains to resected intestinal fragments and components of the extracellular matrix. Arch Microbiol 191, 153161.

Navarre, W. W. \& Schneewind, O. (1999). Surface proteins of Grampositive bacteria and mechanisms of their targeting to the cell wall envelope. Microbiol Mol Biol Rev 63, 174-229.

Oh, P. L., Benson, A. K., Peterson, D. A., Patil, P. B., Moriyama, E. N., Roos, S. \& Walter, J. (2010). Diversification of the gut symbiont Lactobacillus reuteri as a result of host-driven evolution. ISME J 4, 377-387.

Ouwehand, A. C., Tuomola, E. M., Tölkkö, S. \& Salminen, S. (2001). Assessment of adhesion properties of novel probiotic strains to human intestinal mucus. Int J Food Microbiol 64, 119-126.

Pretzer, G., Snel, J., Molenaar, D., Wiersma, A., Bron, P. A., Lambert, J., de Vos, W. M., van der Meer, R., Smits, M. A. \& Kleerebezem, M. (2005). Biodiversity-based identification and functional characterization of the mannose-specific adhesin of Lactobacillus plantarum. J Bacteriol 187, 6128-6136.

Reuter, G. (2001). The Lactobacillus and Bifidobacterium microflora of the human intestine: composition and succession. Curr Issues Intest Microbiol 2, 43-53.

Rinkinen, M., Westermarck, E., Salminen, S. \& Ouwehand, A. C. (2003). Absence of host specificity for in vitro adhesion of probiotic lactic acid bacteria to intestinal mucus. Vet Microbiol 97, 55-61.

Roos, S. \& Jonsson, H. (2002). A high-molecular-mass cell-surface protein from Lactobacillus reuteri 1063 adheres to mucus components. Microbiology 148, 433-442.

Roos, S., Lindgren, S. \& Jonsson, H. (1999). Autoaggregation of Lactobacillus reuteri is mediated by a putative DEAD-box helicase. Mol Microbiol 32, 427-436.

Rosander, A., Connolly, E. \& Roos, S. (2008). Removal of antibiotic resistance gene-carrying plasmids from Lactobacillus reuteri ATCC 55730 and characterization of the resulting daughter strain, L. reuteri DSM 17938. Appl Environ Microbiol 74, 6032-6040.

Salzman, N. H., de Jong, H., Paterson, Y., Harmsen, H. J., Welling, G. W. \& Bos, N. A. (2002). Analysis of $16 \mathrm{~S}$ libraries of mouse gastrointestinal microflora reveals a large new group of mouse intestinal bacteria. Microbiology 148, 3651-3660.

Schreiber, O., Petersson, J., Phillipson, M., Perry, M., Roos, S. \& Holm, L. (2009). Lactobacillus reuteri prevents colitis by reducing P-selectin-associated leukocyte- and platelet-endothelial cell interactions. Am J Physiol Gastrointest Liver Physiol 296, G534G542.

Tallon, R., Arias, S., Bressollier, P. \& Urdaci, M. C. (2007). Strain- and matrix-dependent adhesion of Lactobacillus plantarum is mediated by proteinaceous bacterial compounds. J Appl Microbiol 102, 442451.

Ton-That, H., Marraffini, L. A. \& Schneewind, O. (2004). Protein sorting to the cell wall envelope of Gram-positive bacteria. Biochim Biophys Acta 1694, 269-278.

Turnbaugh, P. J., Hamady, M., Yatsunenko, T., Cantarel, B. L., Duncan, A., Ley, R. E., Sogin, M. L., Jones, W. J., Roe, B. A. \& other 
authors (2009). A core gut microbiome in obese and lean twins. Nature 457, 480-484.

Uchida, H., Fujitani, K., Kawai, Y., Kitazawa, H., Horii, A., Shiiba, K., Saito, K. \& Saito, T. (2004). A new assay using surface plasmon resonance (SPR) to determine binding of the Lactobacillus acidophilus group to human colonic mucin. Biosci Biotechnol Biochem 68, 10041010.

Vesterlund, S., Karp, M., Salminen, S. \& Ouwehand, A. C. (2006). Staphylococcus aureus adheres to human intestinal mucus but can be displaced by certain lactic acid bacteria. Microbiology 152, 18191826.

Wadström, T., Andersson, K., Sydow, M., Axelsson, L., Lindgren, S. \& Gullmar, B. (1987). Surface properties of lactobacilli isolated from the small intestine of pigs. J Appl Bacteriol 62, 513-520.
Walter, J. (2008). Ecological role of lactobacilli in the gastrointestinal tract: implications for fundamental and biomedical research. Appl Environ Microbiol 74, 4985-4996.

Walter, J., Hertel, C., Tannock, G. W., Lis, C. M., Munro, K. \& Hammes, W. P. (2001). Detection of Lactobacillus, Pediococcus, Leuconostoc, and Weissella species in human feces by using group-specific PCR primers and denaturing gradient gel electrophoresis. Appl Environ Microbiol 67, 2578-2585.

Walter, J., Schwab, C., Loach, D. M., Gänzle, M. G. \& Tannock, G. W. (2008). Glucosyltransferase A (GtfA) and inulosucrase (Inu) of Lactobacillus reuteri TMW1.106 contribute to cell aggregation, in vitro biofilm formation, and colonization of the mouse gastrointestinal tract. Microbiology 154, 72-80.

Edited by: V. Eijsink 